Research article

\title{
Distribution pattern and multifarious use of weeds in rice agro- ecosystems of Bhadrak district, Odisha, India
}

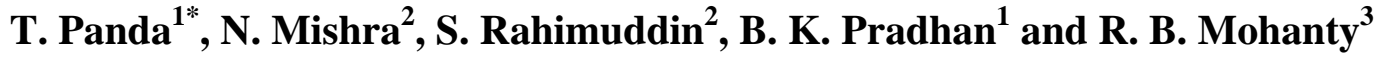 \\ ${ }^{1}$ Chandbali College, Chandbali, Department of Botany, Chandbali- 756133, Odisha, India \\ ${ }^{2}$ Chandbali College, Chandbali, Department of Zoology, Chandbali- 756133, Odisha, India \\ ${ }^{3}$ Ex-Reader in Botany, Plot No. 1311/7628, Satya Bihar, Rasulgarh, Bhubaneswar-751010, Odisha, India
}

*Corresponding Author: taranisenpanda@yahoo.co.in

[Accepted: 20 October 2019]

\begin{abstract}
The weed flora associated with field crop of rice in Bhadrak district of Odisha, India is studied for a period of 2 years (June 2016 to May 2018) based on data obtained from field exploration and literature consultations. Data are collected using standard procedures. The weed association is comprised of 149 species related to 41 angiosperm families and one pteridophytic family. Angiosperms are distributed in 8 superorders and 19 orders. 36.5\% of the species are recorded from the superorder Commelinids, $18.9 \%$ from Malvids, $14.9 \%$ from Lamids, $13.5 \%$ from Fabids and 10.1\% from Companulids as per APG III classification. Order Poales (48), Gentianales and Asterales (14) each, Caryophyllales (13) and Fabales (11) accounts for about $67.6 \%$ of the species in the district. The predominant families are Poaceae and Cyperaceae. The dominant species are Ammannia baccifera, Alternanthera sessilis, Argemone mexicana, Croton sparsiflorus, Cyperus alopecuroides, Echinochloa crusgalli, Eleocharis dulcis, Fimbristylis miliacea, Hygrophila auriculata, Ludwigia hyssopifolia and Oryza rufipogon. Forty eight of the identified species are perennial and 101 annual species. The therophytes are represented by $45.6 \%$ of the life form spectrum. Habitat wise distribution analysis shows forbs share 88 species followed by grasses (29 species) and sedges (18 species) respectively. Of the reported species $37.6 \%$ are used for animal fodder, $29.5 \%$ for the treatment of various diseases and $7.4 \%$ for edible purposes. Considering the importance of weed flora in the rice fields, this research will hopefully improve the monitoring and management of weed flora in the study area and highlight their importance in maintaining ecosystem services which could be a valuable tool for its conservation, preservation and addition of the local biodiversity.
\end{abstract}

Keywords: Agroecosystem - Artifact - Biodiversity - Rice weed - Fodder - Traditional medicines.

[Cite as: Panda T, Mishra N, Rahimuddin S, Pradhan BK \& Mohanty RB (2019) Distribution pattern and multifarious use of weeds in rice agro-ecosystems of Bhadrak district, Odisha, India. Tropical Plant Research 6(3): 345-364]

\section{INTRODUCTION}

Rice (Oryza sativa L.) is one the world's most important staple food crops and a primary food for more than a third of the world's population and has fed more people longer than any other crop, mainly in the tropics (Kumar \& Ladha 2011, Mulungu 2011). In Asia, it is the main diet of 3.5 billion people and most of the countries are highly dependent on their rice yields to meet the increasing demands for food and economical security; thereby the rapidly increasing population in this region is still expected to need $70 \%$ additional rice by 2025 (Kim \& Krishnan 2002). It is also the major source of income and employment for more than 100 million households in Asia and Africa (FAO 2004). The most frequent crop cultivated in India is rice which covers about $25 \%$ of the gross sown area (Singh 1974). The rice supports the local population of 1.25 billion besides other millions of people in Asian and African countries by way of exports (Bumeya \& Ramanathan 2014). Odisha is a leading rice-growing state of the country with rich variety of rice cultivars. The state has 0.44 million hectares of land with this crop, producing 10,322,325 tons of paddy per year (Anonymous 2010). 
According to Yaherwandi (2009), the agricultural landscape consists of paddy ecosystem and weeds. Plants are often called weeds when they opportunistically colonize and occupy habitats artificially disrupted and maintained by humans, e.g., agricultural fields, lawns, and gardens (Baker 1974). About 8000 species have been documented as weeds throughout the globe (Holm et al. 1977). Of these, about 200 species account for approximately 95\% of weed problems in agriculture (Holm et al.1977, 1997). There is little doubt that weeds cause severe economic losses (Chandler \& Cooke 1992) and pose a major threat to world agriculture by reducing detrimentally crop yield and quality by competing with cultivated crops for nutrients, soil, moisture and sunlight (Nyarko \& Datta 1993, Pane et al. 2000). Moreover, weeds can significantly influence crop disease incidence by acting as vectors or reservoirs of plant pathogens (Wisler \& Norris 2005). However, their positive effects cannot be ignored. Weeds have numerous interactions with other organisms and some of these interactions can have direct impacts on the functioning of the agro-ecosystem (Marshall et al. 2003).

Rice fields provide various ecosystem services, such as provisioning of the rice grain, rice straw, and other plant species (i.e. weeds) and animals; cultural services of festivals and rituals associated with farming; supporting nutrient cycles and preserving genetic diversity (Zedler \& Kercher 2005). Datta \& Banerjee (1978), Yamaguchi \& Umemoto (1996), Backman \& Tiainen (2002) and Gibbons et al. (2006) have focused on weeds on paddy crops and pointed out their various functions for food, medicine, prevention of soil erosion, and livestock feed. Flowers of weed can provide alternative feed and as the refuge for insects. Most insect predators and parasitoids get benefit from weeds. Ikeda \& Miura (2002) underlined the major contribution of traditional agricultural practices that supports many endangered wetland plants in paddy fields. Bastiaans et al. (2000) concluded that weeds form the basis of the agro-ecosystem food web. Moreover, the ecology and conservation of wetlands has received much attention (Gopal \& Sah1995). Currently, the number of weeds has been declining drastically in farmed landscapes due to environmental change and anthropogenic activities (Hyvonen 2007, Fried et al. 2009). Thus, there is an urgent need to carry out floristic surveys in rice fields, which harbor many wetland plants, especially in regions where the flora is not well documented.

The composition of the weed flora may differ depending on location, water supply, cultural practices, the inherent weed flora in the area, and the crop grown (Janiya \& Moody 1983, Bhan 1983, Bernasor \& De Datta 1983, Mabbayad et al. 1983, Janiya \& Moody 1989). There have been several reports on the plant species composition of rice fields in Asia including India (Heckman 1974, Chandrasena 1988, Moody 1989, Hakansson 2003, Singh et al. 2008, Chang et al. 2009, Khaliq \& Matloob 2011, Hakim et al. 2013, Mardiyanti et al. 2013, Natuhara 2013, Yun-He \& Sheng 2014, Parameswaran et al. 2014, Fried et al. 2017). A perusal of literature reveals that the knowledge of rice weed communities, their diversity, composition, and distribution in Odisha is inadequate (Satapathy et al. 2012). This study was performed in the Bhadrak district of Odisha, India, where rice fields were the predominant land use. The purposes of this study are two fold: (1) to create an inventory of and classify rice crop weeds and (2) to describe multifarious use of weeds by the local people.

\section{MATERIALS AND METHODS}

Study site

Bhadrak district $\left(20^{\circ} 43^{\prime}-21^{\circ} 13^{\prime} \mathrm{N}\right.$ and $\left.86^{\circ} 6^{\prime}-87^{\circ} \mathrm{E}\right)$ is located in northeast Odisha, India and covers an area of $2505 \mathrm{~km}^{2}$, with a population of 1.507 million (2011 Census). It is bordered by Balasore district in the north, Jajpur in the south, Bay of Bengal and Kendrapara district in the east and Koenjhar in the west. The district accounts for $1.61 \%$ of the state's territory and shares $3.62 \%$ of the state's population. The climate of the region is generally hot and humid with May being the hottest month. Three distinct annual seasons are the rainy season (mid-June to mid-October), winter (mid-October to February) and summer (March to mid-June). The maximum and minimum temperatures range from $37.4^{\circ} \mathrm{C}$ to $17.7^{\circ} \mathrm{C}$ respectively and the annual average rainfall is approximately $1428 \mathrm{~mm}$ (Anonymous 2009, Anonymous 2016). Being located near Bay of Bengal, the district is characterized by periodic earth tremors, rain with thunderstorms and dust storms during April and May (Fig. 1).

The area basically is comprised of deltas formed by two major rivers namely Salandi and Baitarani and half a dozen of rivers and rivulets. Thus the whole district is a fertile monotonous alluvial plain that slopes from west to east following the river gradients. The plain is usually intersected by its own set of rivers and marshland can be seen here and there prominently close to sea line. The soil in Bhadrak district are classified into four categories viz. matured, red \& lateritic soil (alfisols), mixed grey soil (inceptisols), unaltered soil with coarse parent materials (entisols) and unclassified soil (with mudflats). Among all these, inceptisols are found to be the most dominant group of soil in most of the blocks of district (Dhamnagar, Tihidi, Chandbali, Bonth, Bhadrak 
and Basudevpur) except Bhandaripokhari block where alfisols are the dominant soil group. The rice production of the district is 514113.8 tons (Anonymous 2009, Anonymous 2016).

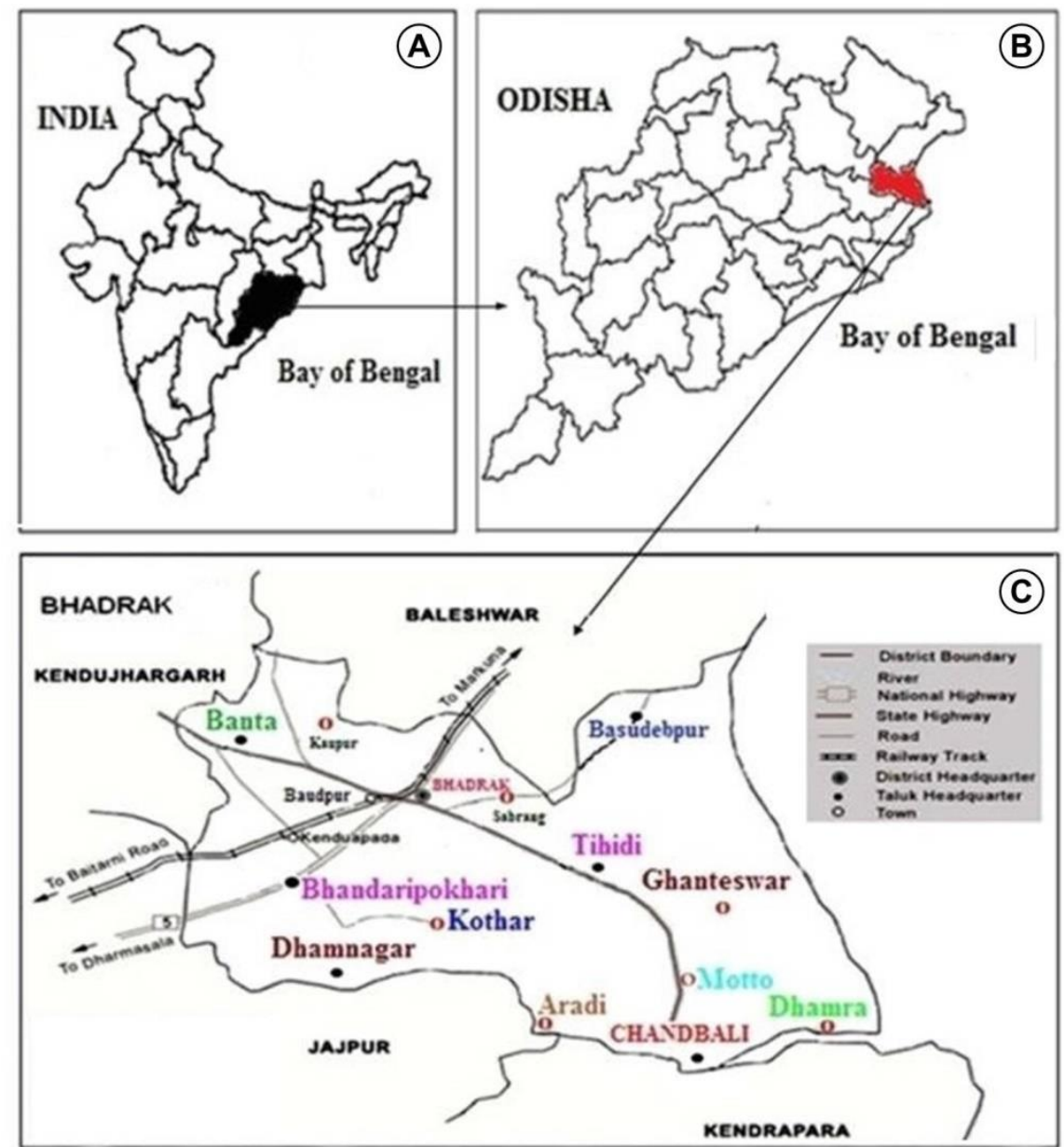

Figure 1. A, Location of Odisha state in the eastern region of India; B, Map of Odisha state showing Bhadrak district; C, Study area showing different blocks of the Bhadrak district.

\section{Data Collection}

The methods employed in this study were designed with the purpose of providing a base line information on the distribution and use of weed plant species through literature survey and field visits to various areas (seven blocks of the district i.e. Basudevpur, Bhadrak, Bhandaripokhari, Bonth, Chandbali, Dhamnagar and Tihidi) from June 2016 to May 2018 in Bhadrak district, Odisha, India. A total of 42 sample plots (29 plots in autumnripening fields, and 13 plots in summer-ripening fields) covering all the blocks were selected. The field study was carried out monthly following established and standard procedures (Jain 1987, Martin 1995). In this investigation, weed species were collected in rice field proper and the bund (levee). The information on the use of weed plants if any was obtained through questionnaires, complemented by free interviews and informal conversations (Martin 1995, Huntington 2000). Elderly farmers in each village were considered the key informants, and the selection process was based on the knowledge base, experience, and current agricultural practices. The interviews and discussions were carried out individually as well as in groups with members of the local population in the local language for each of the villages visited. Personal interviews and group discussions with local inhabitants revealed some valuable and specific information about the plants, which were further compared and authenticated by crosschecking (Cunningham 2001). Eighty-nine (77 men and 12 women) persons were interviewed. Interviews with people were conducted to know local names and uses of sampled plants if any as well as the rice cropping system (cropping seasons, fertilizer application, water management, weed control, yield, grazing after harvest). The collected specimens were processed, dried and herbarium specimens were prepared. All the voucher specimens were maintained in the herbarium of Chandbali College, Chandbali. The plant species are enumerated and arranged as per Angiosperm Phylogeny Group III Classification (APG III 2009). The consulted literatures during field time for identification of species were Haines (1925) and Saxena \& Brahmam (1996). All the plant species were classified on the basis of life forms as defined by Raunkiaer (1934). 


\section{RESULTS}

In the present study, altogether 149 weed species belonging to 41 families and 112 genera from angiosperm and one pteridophyte family were recorded from different rice fields of Bhadrak district (Table 1; Figs. 2-7). The angiosperm taxa were distributed in 112 genera.These taxa were grouped under 9 superorders and 19 orders. 36.5\% of the species were reported from the superorder Commelinids, $18.9 \%$ from Malvids, $14.9 \%$ from Lamids, $13.5 \%$ from Fabids, and 10.1\% from Commelinids as per APG III classification (Fig. 8). Order Poales (48), Gentianales and Asterales (14) each, Carylophyllales (13) and Fabales (11) shared for about 67.6\% of the species in the district. The families Poaceae, Cyperaceae, Asteraceae, Fabaceae and Amaranthaceae were well represented. Commelinaceae, Euphorbiaceae, Onagraceae, Cleomaceae, Malvaceae, Convolvulaceae, Lythraceae, Rubiaceae, Acanthaceae and Scrophulariaceae were moderately represented, while other families between one and two species. The foremost species recorded were Aeschynomene aspera L., Alternanthera sessilis (L.) R.Br. ex DC., Ammannia baccifera L., Argemone mexicana L., Centella asiatica (L.) Urb., Corchorus olitorius L., Croton sparsiflorus Morong., Cyperus alopecuroides Rottb., Cyperus difformis L., Cyperus iria L., Cyperus rotundus L., Desmodium triflorum (L.) DC., Echinochloa crusgalli (L.) P. Beauv., Echinochloa colona (L.) Link., Eleusine indica (L.) Gaertn., Eclipta prostrata (L.) L., Fimbristylis dichotoma (L.) Vahl, Fimbristylis miliacea (L.) Vahl, Hygrophila auriculata (Schum.) Heine., Hydrolea zeylanica (L.) Vahl, Ipomoea aquatica Forssk., Ischaemum rugosum Salisb., Lindernia antipoda (L) Alston., Ludwigia hyssopifolia (G.Don) Exell, Ludwigia perennis L. Murdannia nudiflora (L.) Brenan, Oryza rufipogon Griff., Oxalis corniculata L., Scirpus articulatus L. and Scoparia dulcis L. With respect to life span, annual weeds with 101 species were the most diverse and the remaining were perennials. The therophytes were the most dominant class with 68 plant species $(45.6 \%)$ followed by chamaephytes with 27 species $(18.1 \%)$, hydrophytes with 20 species(13.4\%), cryptophytes and hemicryptophytes with 15 species each (10.1\%), helophytes with 3 species $(2 \%)$ and phanerophytes having one species $(0.7 \%)$ respectively.

Table 1. List of weed flora recorded from Bhadrak district, arranged according to the Angiosperm Phylogeny Group Classification III.

\begin{tabular}{|c|c|c|c|c|c|c|}
\hline Superorder/Order & Family / Species & $\begin{array}{l}\text { Common } \\
\text { Name }\end{array}$ & Habit & $\begin{array}{l}\text { Life } \\
\text { Span }\end{array}$ & $\begin{array}{l}\text { Life } \\
\text { Form }\end{array}$ & Uses \\
\hline \multicolumn{7}{|c|}{ EARLY ANGIOSPERMS } \\
\hline \multirow{3}{*}{ Nymphaeales } & Nymphaeaceae & & & & & \\
\hline & Nymphaea nouchali Burm. f. & Kain & Forb & $\mathrm{P}$ & Hyd & $\begin{array}{l}\text { Medicinal, } \\
\text { edible }\end{array}$ \\
\hline & Nymphaea pubescens Willd. & Rangakain & Forb & $\mathrm{P}$ & Hyd & Edible \\
\hline \multicolumn{7}{|l|}{ MONOCOTS } \\
\hline \multirow[t]{6}{*}{ Alismatales } & Aponogetonaceae & & & & & \\
\hline & $\begin{array}{l}\text { Aponogeton natans }(\text { L.) Engl. \& } \\
\text { Krause }\end{array}$ & Jhechu & Forb & $\mathrm{P}$ & Hyd & Edible \\
\hline & $\begin{array}{l}\text { Aponogeton undulatus Roxb. } \\
\text { Araceae }\end{array}$ & & Forb & $\mathrm{P}$ & Hyd & Edible \\
\hline & $\begin{array}{l}\text { Pistia stratiotes } \mathrm{L} . \\
\text { Hydrocharitaceae }\end{array}$ & Borajhanji & Forb & A & Hyd & Medicinal \\
\hline & Hydrilla verticillata (L.f.) Royle. & Chingudiadala & Forb & $\mathrm{P}$ & Hyd & Medicinal \\
\hline & Ottelia alismoides (L.) Pers. & Panikundri & Forb & $\mathrm{P}$ & Hyd & Medicinal \\
\hline \multirow[t]{2}{*}{ Asparagales } & Amaryillidaceae & & & & & \\
\hline & Crinum asiaticum $\mathrm{L}$. & Arsa & Forb & $\mathrm{P}$ & $\mathrm{Cr}$ & Medicinal \\
\hline \multicolumn{7}{|l|}{ COMMELINIDS } \\
\hline \multirow[t]{7}{*}{ Commelinales } & Commelinaceae & & & & & \\
\hline & Commelina benghalensis L. & Kansiri & Forb & A & $\mathrm{Ch}$ & Medicinal \\
\hline & Commelina difusa Burm.f & & Forb & A & $\mathrm{Ch}$ & Medicinal \\
\hline & $\begin{array}{l}\text { Cyanotis axillaris (L) Schult. \& Schult. } \\
\text { f. }\end{array}$ & & Forb & A & $\mathrm{Ch}$ & Fodder \\
\hline & $\begin{array}{l}\text { Murdannia nudiflora (L.) Brenan } \\
\text { Pontederiaceae }\end{array}$ & Kanduli & Forb & A & $\mathrm{Ch}$ & Fodder \\
\hline & Eichhornia crassipes Solms & Bilatidala & Forb & $\mathrm{P}$ & Hyd & Not Known \\
\hline & Monocharia hastata Solm-Laub. & & Forb & $\mathrm{P}$ & Hyd & Edible \\
\hline \multirow[t]{4}{*}{ Poales } & Poaceae & & & & & \\
\hline & $\begin{array}{l}\text { Brachiaria reptans (L.) Garde. \& } \\
\text { Hubb. }\end{array}$ & & Grass & A & $\mathrm{Ch}$ & Fodder \\
\hline & Chloris barbata $\mathrm{Sw}$. & & Grass & $\mathrm{P}$ & Hem & Fodder \\
\hline & Chrysopogon aciculatus (Retz.) Trin. & Guguchia & Grass & $\mathrm{P}$ & $\mathrm{Cr}$ & Medicinal \\
\hline
\end{tabular}




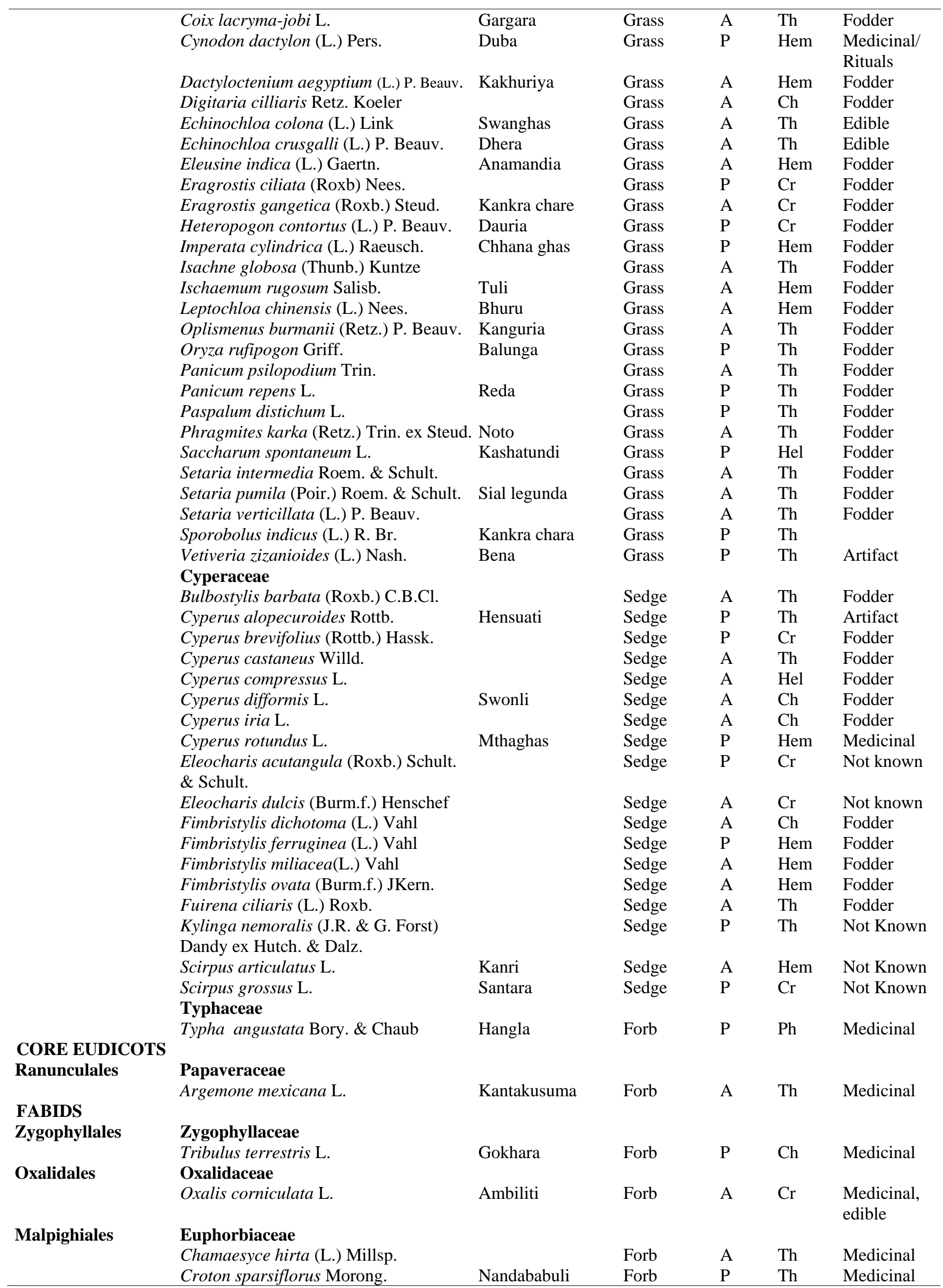




\begin{tabular}{|c|c|c|c|c|c|c|}
\hline & Euphorbia hirta L. & Harharika & Forb & A & $\mathrm{Th}$ & Medicinal \\
\hline & $\begin{array}{l}\text { Euphorbia heterophyla L. } \\
\text { Phyllanthaceae }\end{array}$ & & Forb & A & Th & Not known \\
\hline & Phyllanthus amarus Schum. \& Thonn. & Bhuianla & Forb & A & $\mathrm{Th}$ & Medicinal \\
\hline & $\begin{array}{l}\text { Phyllanthus urinaria } \mathrm{L} . \\
\text { Violaceae }\end{array}$ & Bhuiamla & Forb & A & $\mathrm{Th}$ & Medicinal \\
\hline & Hybanthus enneaspermus (L.) F.v.Muell. & 1. Madan mastak & Forb & A & $\mathrm{Ch}$ & Medicinal \\
\hline \multirow[t]{12}{*}{ Fabales } & Fabaceae & & & & & \\
\hline & Aeschynomene indica $\mathrm{L}$. & Sola & Legume & A & $\mathrm{Cr}$ & Not Known \\
\hline & Aeschynomene aspera $\mathrm{L}$. & Sola & Legume & A & $\mathrm{Cr}$ & Artifact \\
\hline & Alysicarpus monilifer (L.) DC. & & Legume & A & $\mathrm{Th}$ & Fodder \\
\hline & Crotalaria prostrata Rottl. ex Willd. & Jhumka & Legume & $\mathrm{P}$ & $\mathrm{Th}$ & Fodder \\
\hline & Desmodium triflorum (L.) DC. & Kaansisna & Legume & A & $\mathrm{Th}$ & Fodder \\
\hline & Indigofera linnaei Ali & & Legume & $\mathrm{P}$ & $\mathrm{Th}$ & Fodder \\
\hline & Melilotus indica (L.) All & Bana methi & Legume & A & $\mathrm{Th}$ & Fodder \\
\hline & Mimosa pudica L. & Lajakuli & Legume & $\mathrm{P}$ & $\mathrm{Th}$ & Medicinal \\
\hline & Smithia conferta J.E. Sm. & Sanomungo & Legume & A & $\mathrm{Th}$ & Fodder \\
\hline & Tephrosia purpurea (L.) Pers. & Banakolathi & Legume & $\mathrm{P}$ & $\mathrm{Th}$ & Medicinal \\
\hline & Vigna trilobata (L.) Verdc. & & Legume & A & $\mathrm{Cr}$ & Fodder \\
\hline \multicolumn{7}{|c|}{ 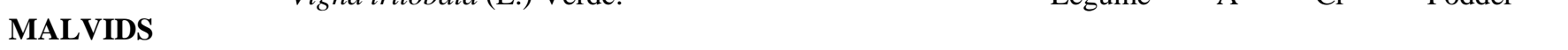 } \\
\hline \multirow[t]{8}{*}{ Myrtales } & Onagraceae & & & & & \\
\hline & Ludwigia adscendens (L.) Hara & Jagal & Forb & A & Hyd & Edible \\
\hline & Ludwigia hyssopifolia (G.Don) Exell & & Forb & A & Hyd & $\begin{array}{l}\text { Medicinal, } \\
\text { edible }\end{array}$ \\
\hline & Ludwigia octovalvis (Jacq.) Raven & & Forb & A & Hyd & Medicinal \\
\hline & $\begin{array}{l}\text { Ludwigia perennis L. } \\
\text { Lythraceae }\end{array}$ & Latkera & Forb & A & Hyd & Not Known \\
\hline & Ammannia baccifera $\mathrm{L}$. & Ramdauni & Forb & A & $\mathrm{Th}$ & Fodder \\
\hline & Ammannia multiflora Roxb. & & Forb & A & $\mathrm{Th}$ & Fodder \\
\hline & Rotala indica (Willd.) Koehne & & Forb & A & $\mathrm{Th}$ & Fodder \\
\hline \multirow[t]{5}{*}{ Brassicales } & Cleomaceae & & & & & \\
\hline & Cleome gynandra L. & Arakasago & Forb & A & $\mathrm{Th}$ & Medicinal \\
\hline & Cleome monophylla $\mathrm{L}$. & Rangasorish & Forb & A & $\mathrm{Th}$ & Not Known \\
\hline & Cleome rutidosperma DC. & & Forb & A & $\mathrm{Th}$ & Medicinal \\
\hline & Cleome viscosa $\mathrm{L}$. & Anasorisho & Forb & A & $\mathrm{Th}$ & Medicinal \\
\hline \multirow[t]{5}{*}{ Malvales } & Malvaceae & & & & & \\
\hline & Corchorus aestuans L. & Bananalita & Forb & A & $\mathrm{Th}$ & Medicinal \\
\hline & Corchorus olitorius L. & & Forb & A & $\mathrm{Th}$ & Not Known \\
\hline & Melochia corchorifolia L. & Telpuri & Forb & $\mathbf{A}$ & $\mathrm{Th}$ & Not Known \\
\hline & Sida acuta Burm.f. & Sunakhadika & Forb & A & $\mathrm{Th}$ & Medicinal \\
\hline \multirow[t]{14}{*}{ Caryophyllales } & Aizoaceae & & & & & \\
\hline & $\begin{array}{l}\text { Trianthema portulacastrum } \mathrm{L} \text {. } \\
\text { Polygonaceae }\end{array}$ & Purinisaga & Forb & A & $\mathrm{Th}$ & Medicinal \\
\hline & $\begin{array}{l}\text { Polygonum plebeium } \mathrm{R} . \mathrm{Br} \text {. } \\
\text { Molluginaceae }\end{array}$ & Muthisaga & Forb & A & Th & Medicinal \\
\hline & Glinus oppositifolius (L.) A. DC. & Pitasaga & Forb & A & $\mathrm{Th}$ & $\begin{array}{l}\text { Medicinal, } \\
\text { edible }\end{array}$ \\
\hline & Amaranthaceae & & & & & \\
\hline & Achyranthes aspera $\mathrm{L}$. & Apamaranga & Forb & A & $\mathrm{Th}$ & Medicinal \\
\hline & Aerva lanata (L.) Juss ex Sch. & Paunsia & Forb & A & $\mathrm{Th}$ & Fodder \\
\hline & $\begin{array}{l}\text { Alternanthera philoxeroides (Mart) } \\
\text { Griseb. }\end{array}$ & & Forb & $\mathrm{P}$ & Hyd & Fodder \\
\hline & Alternanthera sessilis (L.) R.Br. ex DC. & Madranga & Forb & $\mathrm{P}$ & Hyd & $\begin{array}{l}\text { Medicinal, } \\
\text { edible }\end{array}$ \\
\hline & Amaranthus spinosus L. & Kantaneutia & Forb & $\mathrm{P}$ & $\mathrm{Ch}$ & $\begin{array}{l}\text { Medicinal, } \\
\text { edible }\end{array}$ \\
\hline & Celosia argentea $\mathrm{L}$. & Chulia & Forb & A & $\mathrm{Ch}$ & Medicinal \\
\hline & $\begin{array}{l}\text { Gomphrena serrata L. } \\
\text { Portulacaceae }\end{array}$ & & Forb & A & Th & Medicinal \\
\hline & Portulaca oleracea $\mathrm{L}$. & Badabalbaula & Forb & A & $\mathrm{Ch}$ & $\begin{array}{l}\text { Medicinal, } \\
\text { edible }\end{array}$ \\
\hline & Portulaca quadrifida L. & Balbaula & Forb & A & $\mathrm{Ch}$ & Edible \\
\hline
\end{tabular}




\begin{tabular}{|c|c|c|c|c|c|c|}
\hline & $\begin{array}{l}\text { Nyctaginaceae } \\
\text { Boerhavia diffusa } \mathrm{L} .\end{array}$ & Puruni & Forb & $\mathrm{P}$ & $\mathrm{Th}$ & $\begin{array}{l}\text { Medicinal, } \\
\text { edible }\end{array}$ \\
\hline \multicolumn{7}{|l|}{ LAMIDS } \\
\hline \multirow[t]{4}{*}{ Gentianales } & Rubiaceae & & & & & \\
\hline & Dentella repens (L.) J.R. \& Forst. & & Forb & A & $\mathrm{Th}$ & Fodder \\
\hline & Hedyotis corymbosa (L.) Lam. & Jarjati & Forb & A & $\mathrm{Ch}$ & Medicinal \\
\hline & Spermacoce articularis L.f. & Solaganthi & Forb & A & $\mathrm{Ch}$ & Fodder \\
\hline \multirow[t]{12}{*}{ Lamiales } & Acanthaceae & & & & & \\
\hline & Acanthus ilicifolius L. & Harkanch & Shrub & $\mathrm{P}$ & Hel & Medicinal \\
\hline & Hygrophila auriculata (Schum.) Heine. & Koelekha & Forb & $\mathrm{P}$ & $\mathrm{Ch}$ & Medicinal \\
\hline & $\begin{array}{l}\text { Rungia pectinata }(\mathrm{L} .) \text { Nees. } \\
\text { Lamiaceae }\end{array}$ & & Forb & A & Th & Not Known \\
\hline & $\begin{array}{l}\text { Leucas aspera (Willd.) Link. } \\
\text { Scrophulariaceae }\end{array}$ & Gaiso & Forb & $\mathrm{P}$ & $\mathrm{Ch}$ & Medicinal \\
\hline & Bacopa monnieri (L.) Pennell. & Brahmi & Forb & A & $\mathrm{Ch}$ & Medicinal \\
\hline & Lindernia antipoda (L) Alston. & & Forb & A & Th & Fodder \\
\hline & $\begin{array}{l}\text { Lindernia crustacea (L.) F.v.Muell. } \\
\text { Plantaginaceae }\end{array}$ & & Forb & $\mathrm{A}$ & $\mathrm{Th}$ & Not Known \\
\hline & Mecardonia procumbens (Mills.) Small & & Forb & A & $\mathrm{Ch}$ & Not Known \\
\hline & $\begin{array}{l}\text { Scoparia dulcis L. } \\
\text { Verbenaceae }\end{array}$ & Chirarita & Forb & $\mathrm{P}$ & $\mathrm{Ch}$ & Not Known \\
\hline & Phyla nudiflora (L.) Greene & & Forb & A & Th & Fodder \\
\hline & Lippia javanica (Burn.f) Spreng & Naguari & Forb & $\mathrm{P}$ & $\mathrm{Ch}$ & Medicinal \\
\hline \multirow[t]{8}{*}{ Solanales } & Convolvulaceae & & & & & \\
\hline & Evolvulus alsinoides (L.) L. & Bichhamalia & Forb & A & Hem & Medicinal \\
\hline & Evolvulus nummularius (L.) L. & & Forb & A & Hem & Not Known \\
\hline & Ipomoea aquatica Forssk. & Kalamasaga & Climber & $\mathrm{A}$ & Hyd & $\begin{array}{l}\text { Medicinal, } \\
\text { edible }\end{array}$ \\
\hline & $\begin{array}{l}\text { Merremia tridentate (L.) Hall. f. } \\
\text { Hydroleaceae }\end{array}$ & & Climber & A & $\mathrm{Th}$ & Fodder \\
\hline & $\begin{array}{l}\text { Hydrolea zeylanica (L.) Vahl } \\
\text { Sphenocleaceae }\end{array}$ & Languliya & Forb & $\mathrm{P}$ & Hyd & Fodder \\
\hline & $\begin{array}{l}\text { Sphenoclea zeylanica Gaerntn. } \\
\text { Solanaceae }\end{array}$ & Panimircho & Forb & A & Hyd & Fodder \\
\hline & $\begin{array}{l}\text { Solanum surattense Burm. f. } \\
\text { Boraginaceae }\end{array}$ & Beji-begun & Forb & $\mathrm{P}$ & $\mathrm{Th}$ & Medicinal \\
\hline Boraginales & Heliotropium indicum L. & Hatisundha & Forb & A & $\mathrm{Th}$ & Medicine \\
\hline \multirow[t]{15}{*}{$\begin{array}{l}\text { CAMPANULIDS } \\
\text { Asterales }\end{array}$} & Asteraceae & & & & & \\
\hline & Ageratum conyzoides $\mathrm{L}$. & Poksunga & Forb & $\mathrm{A}$ & Th & Medicinal \\
\hline & Blumea membranacea Wall. ex. DC. & Poksunga & Forb & A & $\mathrm{Th}$ & Not Known \\
\hline & Eclipta prostrata $(\mathrm{L}.) \mathrm{L}$. & Bhrungaraj & Forb & $\mathrm{A}$ & Th & Medicinal \\
\hline & Emila sonchifolia (L.) DC. ex Wight. & Sarkara & Forb & A & $\mathrm{Ch}$ & Not Known \\
\hline & Enydra fluctuans Lour. & Hidmichi & Forb & $\mathrm{A}$ & $\mathrm{Cr}$ & Medicinal \\
\hline & Gnaphalium polycaulon Pers. & & Forb & $\mathrm{A}$ & Th & Not Known \\
\hline & Grangea maderaspatana (L.) Poir. & Painjari & Forb & A & $\mathrm{Ch}$ & Not Known \\
\hline & Sphaeranthus indicus L. & Bhuikadamba & Forb & A & Th & Not Known \\
\hline & Spilanthes paniculata Wall. ex. DC. & & Forb & A & $\mathrm{Ch}$ & Fodder \\
\hline & Tridax procumbens $\mathrm{L}$. & Bisalyakarani & Forb & $\mathrm{P}$ & Th & Medicinal \\
\hline & Vernonia cinerea (L.) Less. & Poksunga & Forb & A & Th & Medicinal \\
\hline & $\begin{array}{l}\text { Xanthium indicum Koenig. } \\
\text { Menyanthaceae }\end{array}$ & & Forb & A & $\mathrm{Ch}$ & Not Known \\
\hline & Nymphoides hydrophylla (Lour.) Kuntze & & Forb & $\mathrm{P}$ & Hyd & Edible \\
\hline & Nymphoides indicum (L.) Kuntze & & Forb & $\mathrm{P}$ & Hyd & Edible \\
\hline \multirow[t]{2}{*}{ Apiales } & Apiaceae & & & & & \\
\hline & Centella asiatica (L.) Urb. & Thalkudi & Forb & $\mathrm{A}$ & Hem & $\begin{array}{l}\text { Medicinal, } \\
\text { edible }\end{array}$ \\
\hline Pteridophyte & $\begin{array}{l}\text { Marsileaceae } \\
\text { Marsilea minuta L. }\end{array}$ & & Forb & A & $\mathrm{Cr}$ & Edible \\
\hline
\end{tabular}
Hydrophyte; Th, Therophyte. 

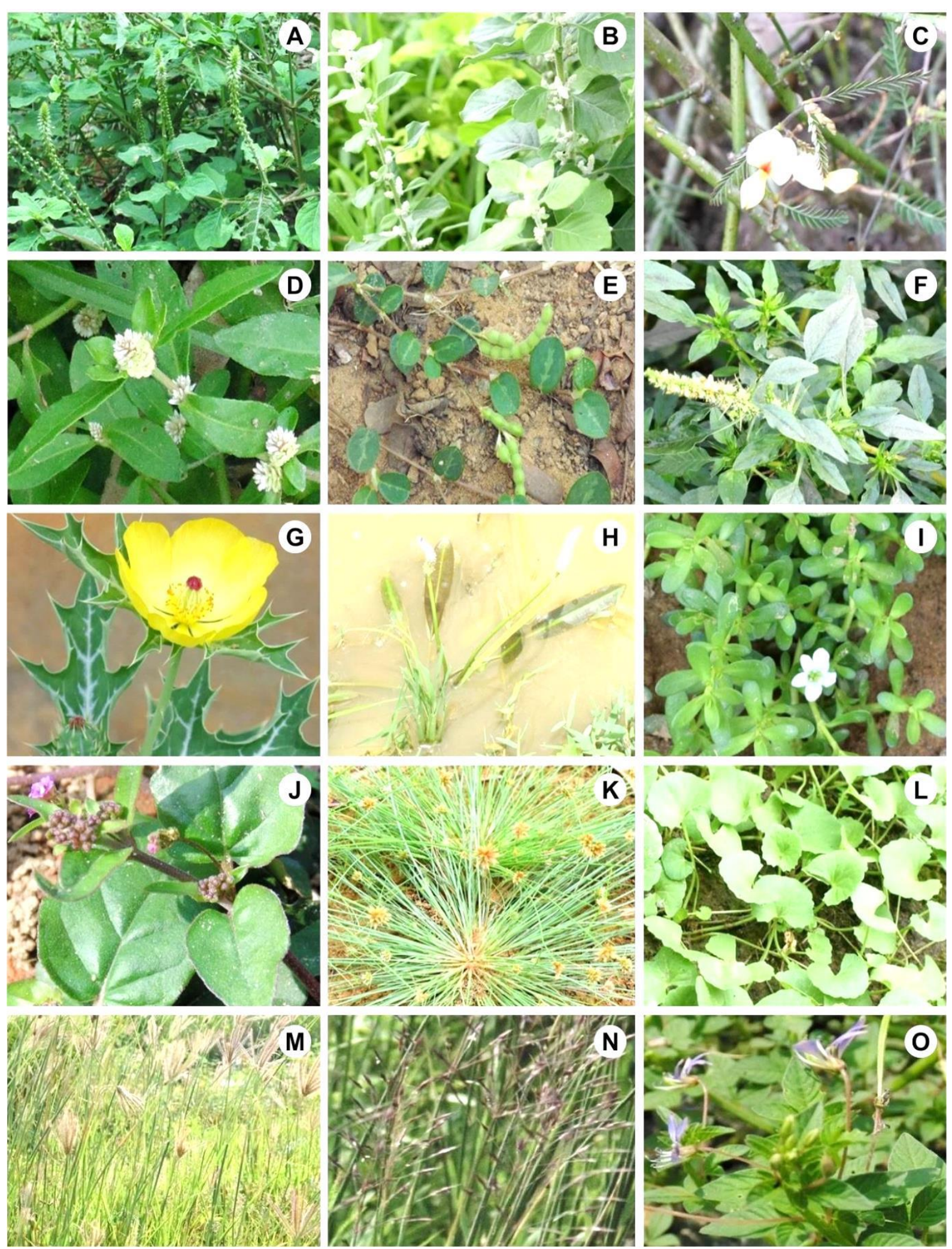

Figure 2. Some plant species observed during the study: A, Achyranthes aspera L.; B, Aerva lanata (L.) Juss ex Sch.; C, Aeschynomene aspera L.; D, Alternanthera sessilis (L.) R.Br. ex DC.; E, Alysicarpus monilifer (L.) DC.; F, Amaranthus spinosus L.; G, Argemone mexicana L.; H, Aponogeton natans (L.) Engl. \& Krause; I, Bacopa monnieri (L.) Pennell.; J, Boerhavia diffusa L.; K, Bulbostylis barbata (Rottb.) C.B.Cl.; L, Centella asiatica (L.) Urban; M, Chloris barbata Sw.; N, Chrysopogon aciculatus (Retz.) Trin.; O, Cleome rutidosperma DC. [Photographs by Taranisen Panda] 

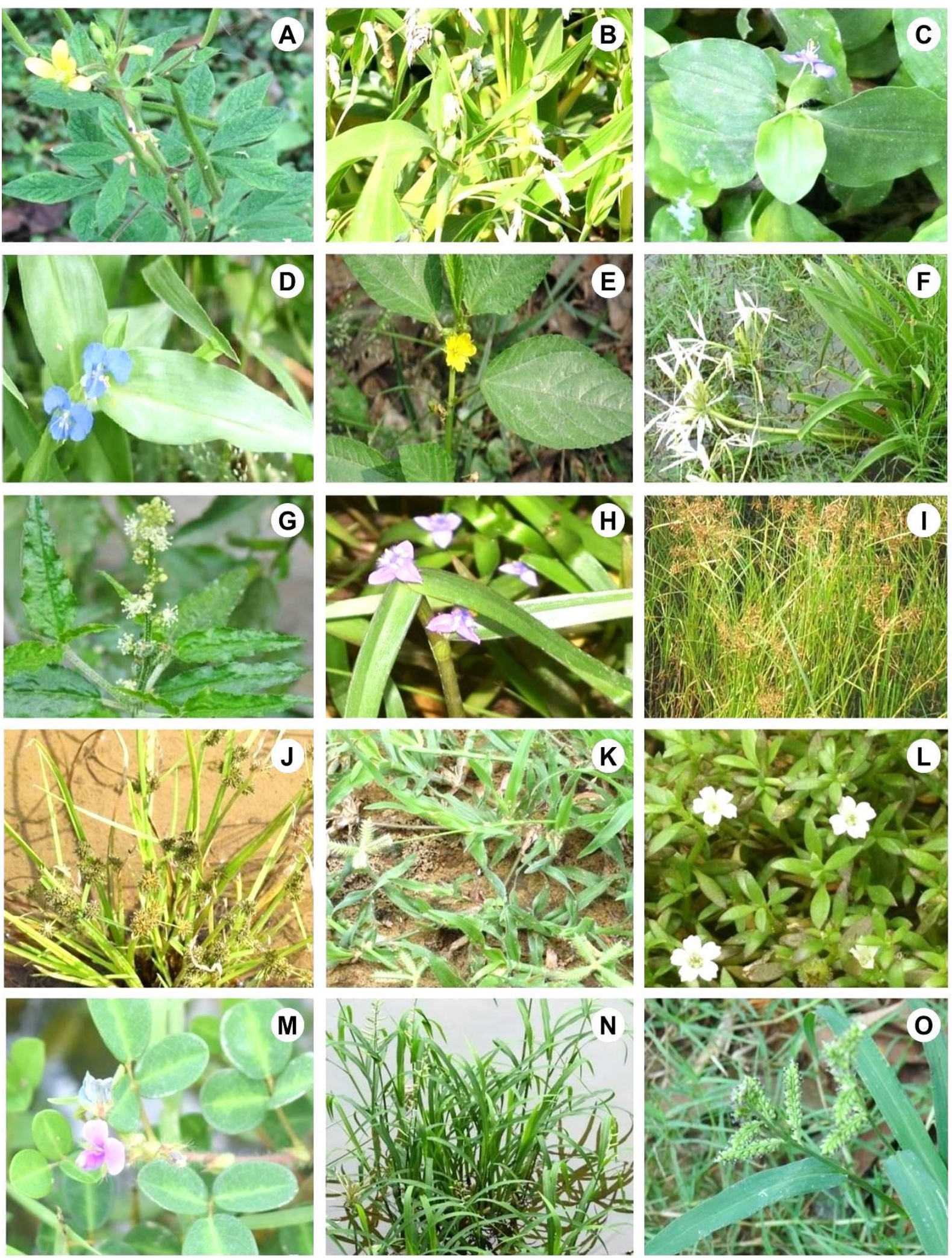

Figure 3. Some plant species observed during the study: A, Cleome viscosa L.; B, Coix lacryma-jobi L.; C, Commelina benghalensis L.; D, Commelina difusa Burm.f.; E, Corchorus olitorius L.; F, Crinum asiaticum L.; G, Croton sparsiflorus Morong.; H, Cyanotis axillaris (L) Schult. \& Schult. f.; I, Cyperus alopecuroides Rottb.; J, Cyperus difformis L.; K, Dactyloctenium aegyptium (L.) P. Beauv.; L, Dentella repens (L.) Forst. et Forst.; M, Desmodium triflorum (L.) DC.; N, Echinochloa colona (L.) Link; O, Echinochloa crusgalli (L.) P. Beauv.;. [Photographs by Taranisen Panda] 

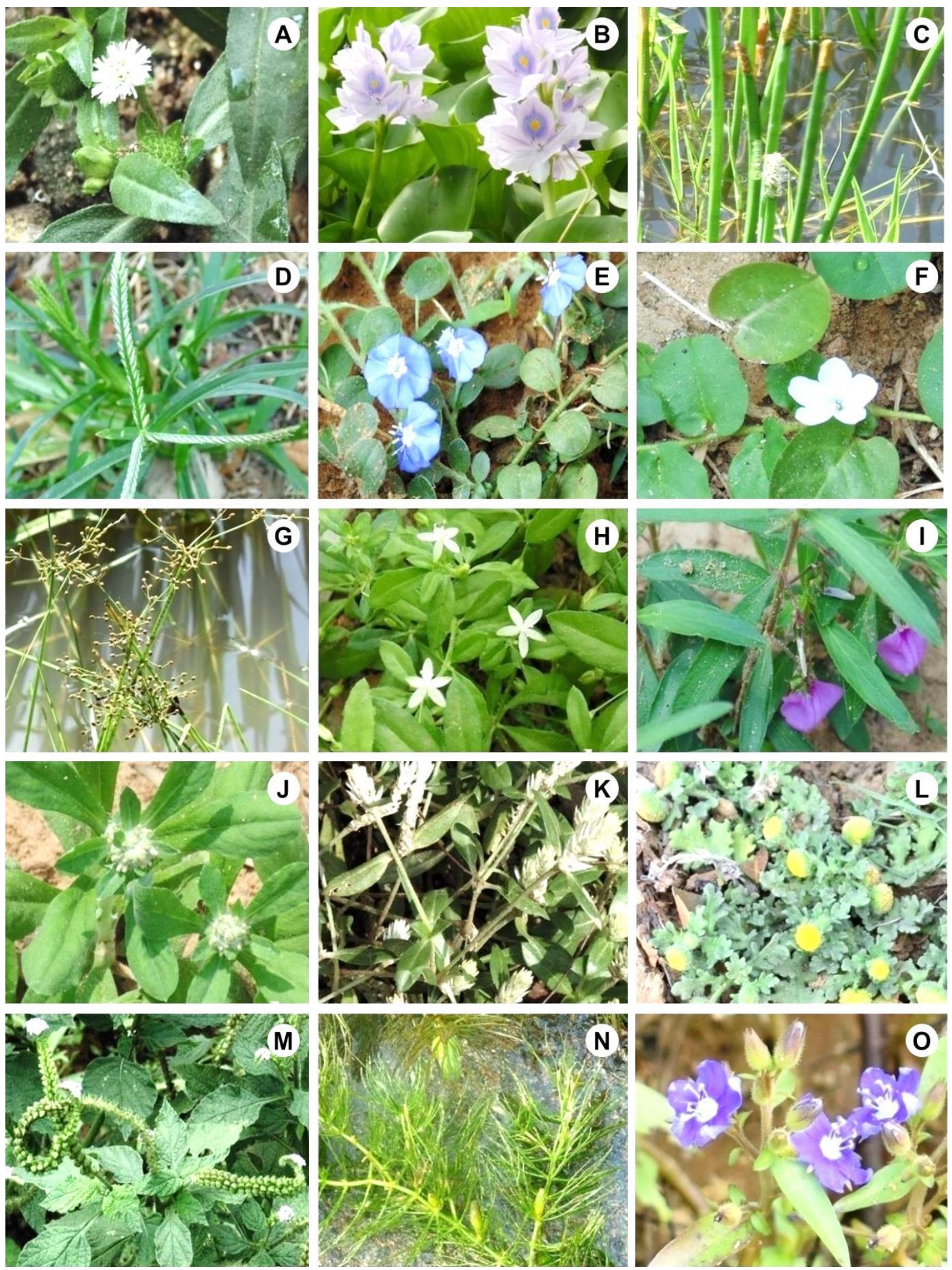

Figure 4. Some plant species observed during the study: A, Eclipta alba (L.) Hassk.; B, Eichhornia crassipes Solms; C,

Eleocharis dulcis (Burm. f) Henschef; D, Eleusine indica (L.) Gaertn.; E, Evolvulus alsinoides (L.) L.; F, Evolvulus nummularius (L.) L.; G, Fimbristylis miliacea (L.) Vahl; H, Hybanthus enneaspermus (L.) F.v. Muell.; I, Glinus oppositifolius (L.) A. DC.; J, Gnaphalium polycaulon Pers.; K, Gomphrena serrata L.; L, Grangea maderaspatana (L.) Poir.; M, Heliotropium indicum L.; N, Hydrilla verticillata (L. f.) Royle.; O, Hydrolea zeylanica (L.) Vahl. [Photographs by Taranisen Panda] 

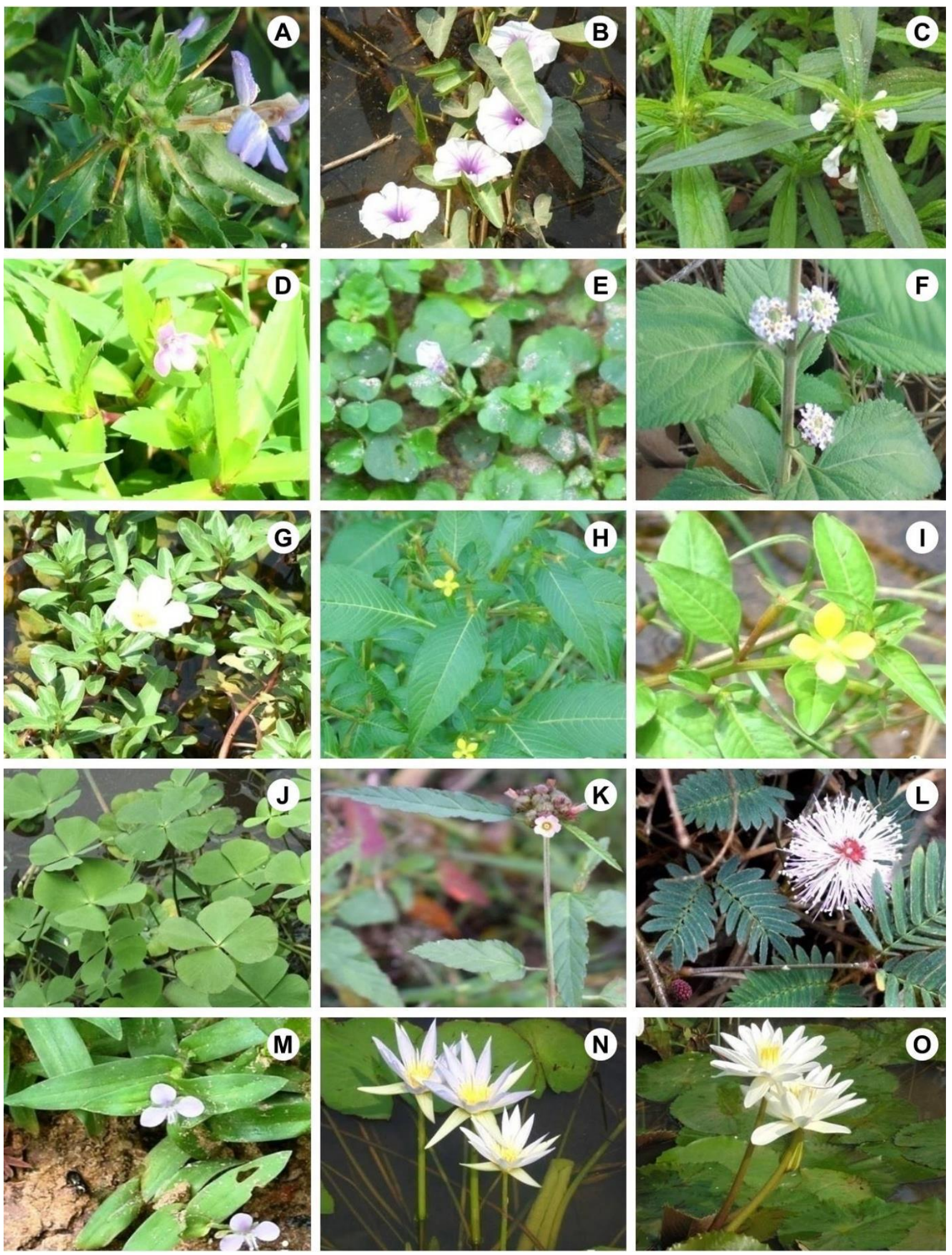

Figure 5. Some plant species observed during the study: A, Hygrophila auriculata (Schum.) Heine.; B, Ipomoea aquatica Forssk.; C, Leucas aspera (Willd.) Link.; D, Lindernia antipoda (L) Alston.; E, Lindernia crustacea (L.) F.v.Muell.; F, Lippia javanica (Burn.f) Spreng; G, Ludwigia adscendens (L.) Hara; H, Ludwigia hyssopifolia (G.Don) Exell; I, Ludwigia perennis L.; J, Marsilea minuta L.; K, Melochia corchorifolia L.; L, Mimosa pudica L.; M, Murdannia nudiflora (L.) Brenan; N, Nymphaea nouchali Burm.f.; O, Nymphaea pubescens Willd. [Photographs by Taranisen Panda] 

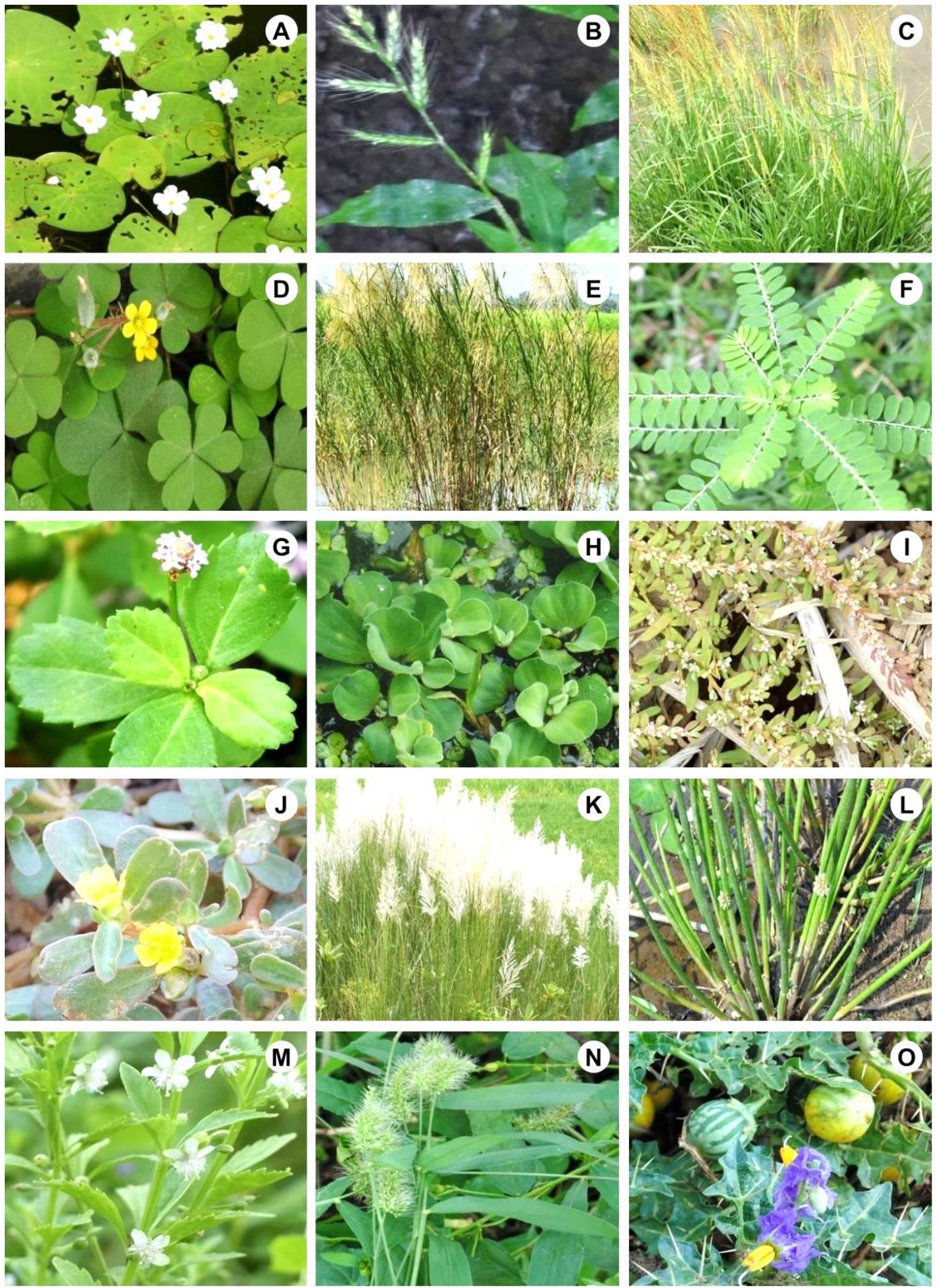

Figure 6. Some plant species observed during the study: A, Nymphoides hydrophylla (Lour.) Kuntze; B, Oplismensus burmanii (Retz.) P. Beauv.; C, Oryza rufipogon Griff.; D, Oxalis corniculata L.; E, Phragmites karka (Retz.) Trin. ex Steud.; F, Phyllanthus amarus Schum. \& Thonn.; G, Phyla nudiflora (L.) Greene; H, Pistia stratiotes L.; I, Polygonum plebeium R.Br.; J, Portulaca oleracea L.; K, Saccharum spontaneum L.; L, Scirpus articulatus L.; M, Scoparia dulcis L.; N, Setaria verticillata (L.) P. Beauv.; O, Solanum surattense Burm. f. [Photographs by Taranisen Panda] 

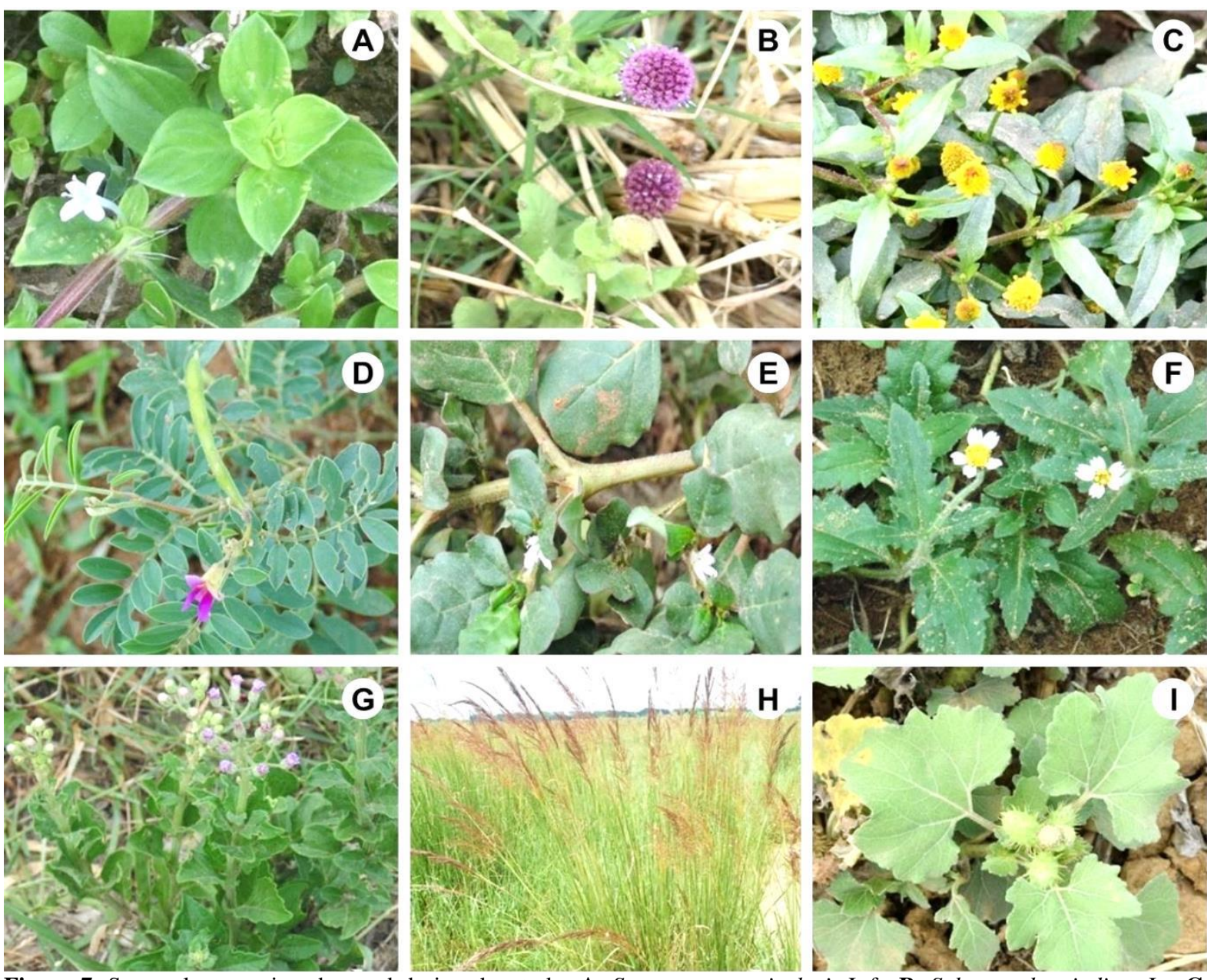

Figure 7. Some plant species observed during the study: A, Spermacoce articularis L.f.; B, Sphaeranthus indicus L.; C, Spilanthes paniculata Wall ex DC.; D, Tephrosia purpurea (L.) Pers.; E, Trianthema portulacastrum L.; F, Tridax procumbens L.; G, Vernonia cinerea (L.) Less.; H, Vetiveria zizanioides (L.) Nash.; I. Xanthium indicum Koenig. [Photographs by Taranisen Panda]

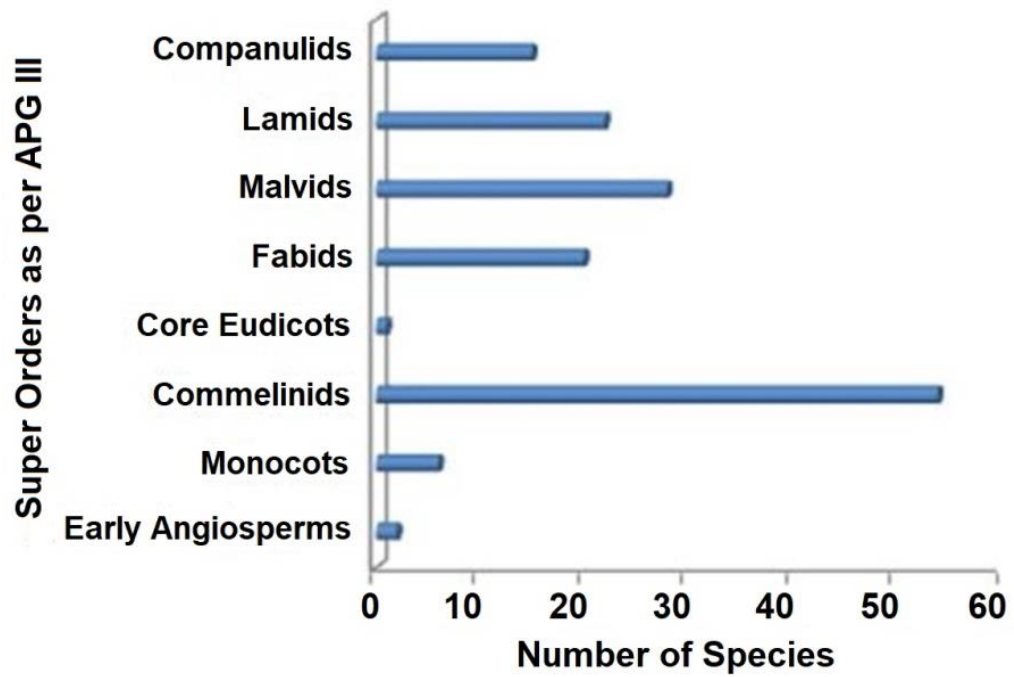

Figure 8. Distribution of species in super order as per APG III.

In terms of multifarious use of weeds (Fig. 9), 37.6\% of the recorded species were used for animal fodder, $29.5 \%$ for the treatment of various diseases and $7.4 \%$ for edible purposes. Habitat wise distribution analysis (Fig. 10) showed forbs share 88 species followed by grasses ( 29 species) and sedges (18 species) respectively.

\section{DISCUSSION}

The abundance and distribution of weed species within the landscape of the rice field play pivotal role for maintenance of biodiversity. The years of agricultural practices have coevolved with the environment, and the resulting interactions brought forth local wisdom in rice farming practices that has also influenced and shaped www.tropicalplantresearch.com 
the cultural heritage of the society (Norgaard 1984). Farmers experiment and innovate new methods in an uxexpectrd way on trial and error method to improve their livelihood without any policy of the government (Dey 2012). However, through modern agricultural methods, higher yields and greater food security have come by degrading environment, reducing interspecific competition and enhancing biodiversity loss. Therefore by adopting alternative sustainable agriculture practices the diversity of paddy ecosystem can be protected (Chappell \& Lavalle 2011). The number of weed species recorded from Bhadrak district is comparable with other regions of Asia [191 species in Japan (Kasahara 1959),136 species in Sri Lanka (Chandrasena 1988), 96 species in Thailand (Tomita et al. 2003a,b), 145 species in Tamil Nadu, India (Dhanam \& Elayaraj 2014 ), 82 species in Nepal (Nowak et al. 2016), 113 species in Vietnam and the Philippines (Fried et al. 2017), 272 species in China (Yun-he \& Sheng 2014)]. The highest contribution of family Poaceae is in accordance with Turki \& Sheded (2002) and Hakim et al. (2013).

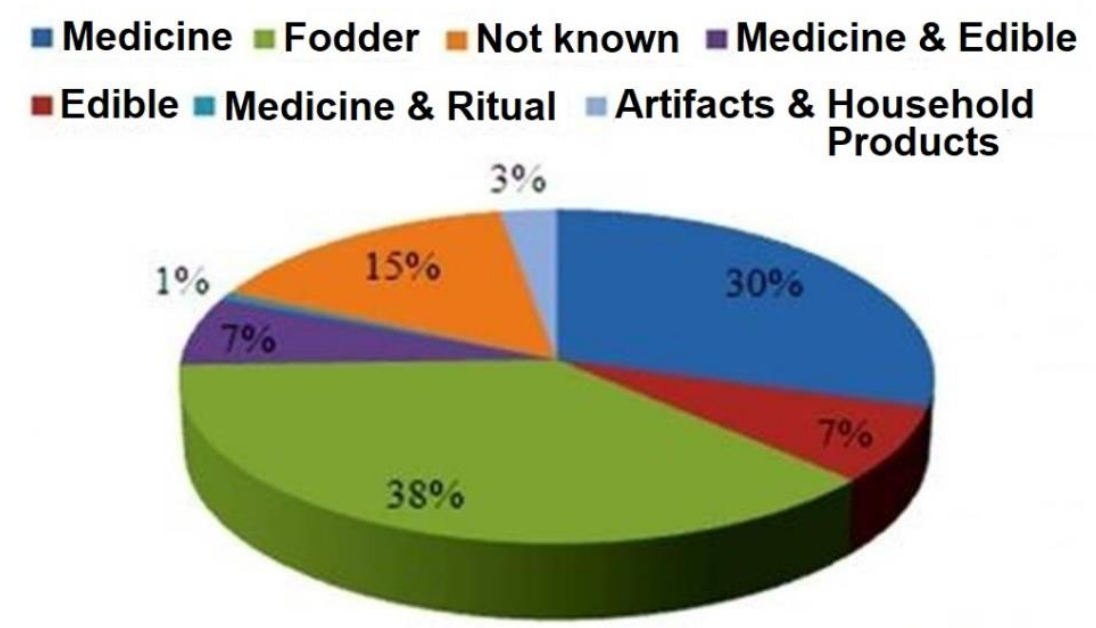

Figure 9. Multifarios use of weed species collected at the study site.

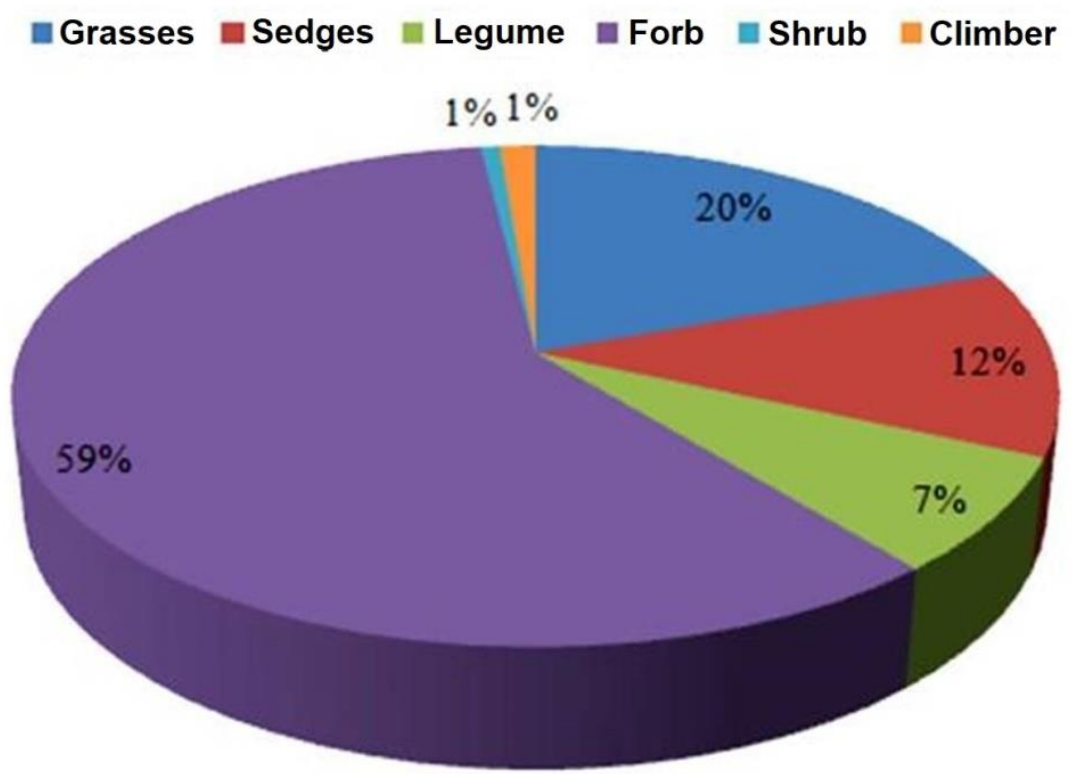

Figure 10. Habitat wise distribution of weed species at the study site.

The species listed in this study are also reported as weeds in other countries (Dekker 1997, Qiang 2002, Bhatt et al. 2009, Hakim et al. 2010, Jaya Suria et al. 2011, Khaliq et al. 2011). In fact, from the recorded 12 species of Asia's worst weed (IRRI 2010), Bhadrak district harbors seven weed species (Cyperus iria, Cyperus difformis L., Echinochloa colona, Ischaemum rugosum, Leptochloa chinensis Nees, Ludwigia hyssopifolia, Sphenochlea zeylanica Gaertn.). Out of the ten major weeds reported from transplanted rice of India (Rao et al. 2017), Bhadrak district represents eight species (Echinochloa colona, Echinochloa crusgalli, Cyperus difformis, Cyperus iria, Fimbristylis miliacea, Eclipta prostrata, Cyperus rotundus, Ammannia baccifera). Individually, the incursion by Cyperus rotundus, Eichhornia crassipes (C. Martius) Solms., Fimbristylis dichotoma and F. miliacea, Cyperus iria and Cyperus difformis are causing great concern in many rice fields of this district. Freshwater species like Eichornia crassipes is of the most nuisance as it causes hindrance by choking all www.tropicalplantresearch.com 
possible water bodies and reducing their utility. Because of its rapid growth rate, water hyacinth is able to outcompete native aquatic plants by utilising the available nutrients in the water, and successfully competing for space and sunlight (Cilliers 1991). Echinochloa crusgalli is one of the widespread weeds of rice globally (Michael 1978). It finds a place on the list of 10 worst weeds of the world (Noda 1977). Echinochloa crusgalli is reported as a dominant weed in rice fields of Malaysia (Hakim et al. 2010), Nepal (Bhatt et al. 2009), Pakistan (Khaliq et al. 2011) and India (Parameswaran et al. 2014). While studying the weed community of Zhejiang Province, southeastern China Yu et al. (1993) stated that Echinochloa crusgalli is the dominant weed species of the early and late rice stages respectively. Famers confuse this weed because it mimics rice plants during the early stage. Cyperus rotundus is considered the world's worst weed because of its ability to survive, spread, and compete, especially in agricultural areas (Holm et al. 1977, Terry 2001). It is reported in 52 crops and 92 countries (Holm et al. 1979, 1997). Their rhizomes form tubers that give rise to new aerial plants or produce other rhizomes or they may remain dormant during periods of adverse environmental conditions including heat, cold, drought, flooding, or inadequate aeration (Wills 1987, Miles et al. 1996). Cyperus difformis and C. iria are ranked $32^{\text {nd }}$ and $33^{\text {rd }}$ among the world's worst weeds, respectively (Holm et al. 1977). They often produce clumps of many culms and have become established in tropical and temperate areas of the world. Fimbristylis dichotoma and F. miliacea are co-ranked as the world's $40^{\text {th }}$ worst complex of weeds (Holm et al. 1977). Fimbristylis dichotoma is a rapidly growing and thrives in poorly aerated soils with high moisture content (Holm et al. 1977). It has been reported as a weed of paddy crops, in 21 countries throughout the tropical and semitropical regions of the world including Africa, Asia, the Pacific Islands, and North and South America (Holm et al. 1977). Nowak et al. (2016) observed the higher occurrence of Alternanthera sessilis, Cyperus difformis, Centella asiatica, Cynodon dactylon (L.) Pers., Echinochloa colona, Fimbristyllis miliacea, Cyperus iria and Marsilea minuta $\mathrm{L}$. in rice field of central Nepal.

Studies of Sharma et al. (2014) and Thakur (2015) have shown that biological spectrum of a region indicates the phytoclimate of that region. Higher percentage of therophytes in the studied area reflects a therophytic phytoclimate of the district. The rich therophytic flora may be due to the grazing, weeding or other anthropogenic interference in the area during the process of cultivation which reduces the number of other life forms. The predominance of annual weed species over the perennial ones is similar to the findings of Shivakumar et al.(2014).

Even though agricultural scientists and extension officers recommend eradication of weeds, $89 \%$ of the most widespread and aggressive weeds in the world are edible (Rapoport et al. 1995). Moreover, many of these species have a high nutritional value and medicinal properties (Duke 1992). Most of the plant species of the study area have important economic uses. Weeds like Amaranthus spinosus L., Echinochloa crusgalli, Glinus oppositifolius (L.) A.DC., Marsilea minuta, Monochoria hastata (L.) Solms, Nymphaea nouchali Burm. f., Nymphaea pubescens Willd., Oxalis corniculata, Portulaca oleracea L. and Portulaca quadrifida L. are used for edible purposes by the local inhabitants. The consumption of weeds is a world-wide phenomenon that is noted as having an important role for human nutrition (Duke 1992, Pemberton et al. 1996, Marcelino et al. 2005, Maneechote 2007, Turner et al. 2011). Some of the edible weed plants like Ipomoea aquatica and Marsilea minuta are collected by local people and sold in the local markets particularly by poor and economically marginalised families, thereby generating a supplementary income.

A number of weeds reported from the study area are used by local people in traditional medicines for their primary health care. For instance, burned root ashes of Achyranthes aspera L. are applied topically to reduce the pain of the skin infected with worms as well as to expel the dead worms out. Warmed leaves of Amaranthus spinosus are applied locally to cure boils and burns. Fresh leaf paste of Argemone mexicana is applied topically to treat eczema. Decoction of whole plant of Boerhavia diffusa $\mathrm{L}$. is used to treat leucorrhoea. Juice of Cynodon dactylon is used to stop nose bleeding. Latex of Euphorbia hirta L. is effective for healing of wounds. Glinus oppositifolius is used either in raw or cooked form to cure various types of skin disease like scabies, itches etc. Mimosa pudica L. roots are chewed for toothache. Rhizome paste of Nymphaea nouchali is administered to regulate menstruation. Tender twig of Phyllanthus amarus Schum. \& Thonn. is used to cure dysentery. Leaf decoction of Tephrosia purpurea L. is prescribed to women against post natal complications. Decoction of Tridax procumbens $\mathrm{L}$. leaf is applied topically on the boils, cuts, sores, wounds and eczema to promote healing. Crushed tuber powder of Cyperus rotundus is taken orally for jaundice. Root bark of Scoparia dulcis is chewed for toothache and dental caries. The present finding draws support from the studies of Mishra (2016) and Parameswaran \& Kumar (2017). A number of weeds such as Alternanthera sessilis, Bacopa monnieri (L.) Pennell., Centella asiatica, Boerhavia diffusa, Commelina benghalensis L., Eclipta prostrata, Enydra fluctuans 
Lour., Hygrophila auriculata are reported to have both therapeutic and dietary functions and hence are used as medicinal food remedy. This overlap indicates the close relationship between health and food. Overlapping between food and medicines is quite well known in traditional societies (Panda \& Misra 2011, Swapna et al. 2011, Mishra et al. 2012). The study area is also considered as important source for animal wellbeing where many weed species are utilized as fodder for buffaloes and cattle (Marcelino et al. 2005).

A good number of artifact items and household articles are prepared from Aeschynomene aspera, Cyperus alopecuroides and Vetiveria zizanioides (L.) Nash. by the artisans of the district. Similar observations have also been made in earlier studies (Mohanty et al. 2012, Tripathy et al. 2014).

The increased frequency of burning crop residues after harvesting caused a dramatic loss of weed biodiversity in January-February. Burning could be an attractive residual management option for farmers to prepare for the subsequent rice cropping. However, burning have the negative aspects such as greenhouse gas emission, nutrient loss, diminished soil biota, and reduced total N and C in the topsoil (Gupta \& Sahai 2005, Wassmann et al. 2009), as many governments in developing Asian countries made it illegal to burn crop residues (Singh et al. 2005). Farmers might lack an understanding of these negative aspects of burning for environment, as well as knowledge of available technology for in situ incorporation of the residues (Samra et al. 2003).

\section{CONCLUSIONS}

Rice fields are an important component of Odishan culture and play a pivotal role in the agricultural ecological system. The results highlight the significant contribution of rice field in conserving biodiversity of this region, since rice fields form the largest freshwater ecosystem of the district. It can be concluded that the vegetation of the agro-climatic zone of Bhadrak district is mostly seasonal and annual weeds predominate, majority of which continue to survive in subsequent periods by their seeds or vegetative propagules. Agricultural chemicals (including pesticides, synthetic fertilizers and growth regulators) have contributed substantially to the development of modern agriculture, but the overuse has also damaged agricultural areas and the surrounding natural vegetation, aquatic environment and wildlife, thus decreasing rice paddy biodiversity whichleads to the unsustainable development of the rice field ecosystem. The conservation of the rice field ecosystem and biodiversity needs to be further strengthened through protecting the ecological environment surrounding the rice fields, improving rice cropping patterns, growing rice with less agricultural chemicals and synthetic fertilizers and promoting ecological education and public awareness and, thus, achieves ecological sustainability.

\section{ACKNOWLEDGEMENT}

The authors are thankful to the villagers for their generous hospitality and sharing their traditional knowledge for documentation and photography.

\section{REFERENCES}

Anonymous (2009) District statistical hand book. Bhadrak. Directorate of economics \& statistics, Bhubaneswar, Odisha.

Anonymous (2010) Districts at a Glance. Bhubaneswar, Odisha.

Anonymous (2016) Proceedings of the $2^{\text {nd }}$ district level implementation committee of the Pradhan Mantri Krushu Sinchayee Jojana of Bhadrak district.

APG III (2009) An update of the angiosperm phylogeny group classification for the orders and families of flowering plants. Botanical Journal of the Linnaean Society 161 (2): 105-121.

Baker HG (1974) The evolution of weeds. Annual Review of Ecology Evolution System 5: 1-24.

Backman JPC \& Tiainen J (2002) Habitat quality of field margins in a Finnish farmland area for bumblebees (Hymenoptera: Bombus and Psithyrus). Agriculture, Ecosystems \& Environment 88: 53-68.

Bastiaans L, Kropff MJ, Goudriaanb J \& Van Laar HH (2000) Design of weed management systems with a reduced reliance on herbicides poses new challenges and prerequisites for modeling crop-weed interactions. Field Crop Research 67: 161-179.

Bernasor PC \& De Datta SK (1983) Integration of cultural management and chemical control on weeds in broadcast seeded flooded rice. In: Proceedings of $9^{\text {th }}$ Asian Pacific Weed Science Society Conference, pp. $137-155$.

Bhan VM (1983) Effect of hydrology, soil moisture regimes, and fertility management on weed populations and their control in rice. In: Weed Control in Rice. Laguna, pp. 47-56. 
Bhatt MD, Tewari A \& Singh SP (2009) Floristic Composition of weeds in paddy fields In Mahendranagar, Nepal. Ecoprint 16: 15-19.

Bumeya J \& Ramanatha V(2014) Recent climate and air pollution impacts on Indian agriculture. PNAS 111: 16319-16324.

Chandler JM \& Cooke FT (1992) Economics of cotton losses caused by weeds. In: Mcwhorter CG \& Abernathy JR (eds) Weeds of Cotton: Characterization and Control. Memphis, pp. 85-116.

Chandrasena JPNR (1988) Floristic composition and abundance of rice-field weeds in four low country wet zone districts of Sri Lanka. Tropical Pest Management 34 (3): 278-287.

Chang X, Li R, Zhu W \& Chen Q (2009) Weed communities and distribution characteristics in the paddy fields of main rice growing regions of Hubei Province. Chinese Journal of Eco-Agriculture 17: 533-536.

Chappell MJ \& Lavalle LA (2011) Food security and biodiversity: can we have both? An agroecological analysis. Agriculture Human Values 28: 3-26.

Cilliers CJ (1991) Biological control of water hyacinth, Eichhornia crassipes (Pontederiaceae), in South Africa. Agriculture Ecosystems \& Environment 37: 207-218.

Cunningham AB (2001) Applied ethnobotany: People, wild plant use and conservation. Earthscan Publishing Ltd., London, UK.

Datta SC \& Banerjee AK (1978) Useful weeds of west Bengal rice fields. Economic Botany 32: 297-310.

Dekker J (1997) Weed diversity and weed management [J]. Weed Science 45(3): 357-363.

Dey MM, Spielman DJ, Mahfuzul Haque ABM, Rahman MS \& Valmonte-Santos RA (2012) Change and diversity in smallholder rice-fish systems: recent evidence from Bangladesh. IFPRI Discussion Paper 01220, Washington.

Dhanam S \& Elayaraj B (2014) Ethnomedicinal aspects of some weeds from paddy fields of Villupuram district in Tamil Nadu, India. International Letters of Natural Sciences 14: 1-10.

Duke JA (1992) Handbook of edible weeds. Boca Raton.

FAO (2004) Rice and us. Food and Agriculture Organization of the United Nations.

Fried G, Petit S, Dessaint F \& Reboud X (2009) Arable weed decline in Northern France: Crop edges as refugia for weed conservation? Biological Conservation 142: 238-243.

Fried O, Kühn I, Schrader J, Van Sinh N \& Bergmeier E (2017) Plant diversity and community composition of rice agroecosystems in Vietnam and the Philippines. Phytocoenologia 47(1): 49-66.

Gibbons DW, Bohan DA \& Rothery P (2006) Weed seed resources for birds in fields with contrasting conventional and genetically modified herbicide-tolerant crops. Philosophical Transactions of Royal Society of London B273: 1921-1928.

Gopal B \& Sah M (1995) Inventory and classification of wetlands in India. Vegetatio 118: 39-48.

Gupta PK \& Sahai S (2005) Residue open burning in rice wheat cropping systemsin India: an agenda for conservation of environment and agricultural resources. In: Abrol IP, Gupta, RK \& Malik RK (eds) Conservation Agriculture Status and Prospects. New Delhi, pp. 50-54.

Haines HH (1925) The Botany of Bihar and Orissa. Adland and Son, West Newman Ltd., London.

Hakim MA, Juraimi AS, Ismail MR, Hanafi MM \& Selamat A (2010) Distribution of weed population in coastal; rice growing area of Kedha in Peninsular Malaysia. Journal of Agronomy 9(1): 9-16.

Hakim MA, Juraimi AS, Ismail MR, Hanafi MM \& Selamat A (2013) A comparison of weed communities of coastal rice fields in Peninsular Malaysia. Journal of Environmental Biology 34: 847-856.

Heckman CW (1974) The seasonal succession of species in a rice paddy in Vientiane, Laos. Internationale Revue Der Gesamten Hydrobiologie 59: 489-507.

Hakansson S (2003) Weeds and weed management on arable land. An ecological approach. Wallingford, p. 288.

Holm LG, Plucknett DL, Pancho JV \& Herberger JP (1977) The World's worst weeds: Distribution and biology. University of Hawaii Press, Honolulu.

Holm LG, Pancho JV, Herberger JP \& Plucknett DL (1979) A Geographical atlas of world weeds. New York.

Holm LG, Doll J, Holm E, Pancho J \& Herberger J (1997) World Weeds: Natural Histories and Distribution. John Wiley \& Sons, Inc., New York.

Hyvonen T (2007) Can conversion to organic farming restore the species composition of arable weed communities. Biological Conservation 137: 382-390.

Huntington HP (2000) Using traditional ecological knowledge in science: Methods and applications. Ecological Applications 10(5): 1270-1274. 
Ikeda R \& Miura R (2002) Tsurugashi nakaikemi no shitsudennoukou to zetsumetsukigu syokubutsu. Noukou no Gijutsu to Bunka 23: 43-72. [in Japanese]

IRRI (2010) Rice knowledge bank. International Rice Research Institute, Makati.

Jain SK (1987) Glimpses of Indian ethnobotany.Oxford \& IBH Publishing Co., New Delhi.

Janiya JD \& Moody K (1983) Weed growth and yield of two rice crops grown in sequence in three rainfed locations in the Philippines. Philipine Agriculture 66: 90-101.

Janiya JD \& Moody K (1989) Weed populations in transplanted and wet-seeded rice as affected by weed control method. Tropical Weed Management 35(1): 8-11.

Jaya Suria ASM, Juraimi AS, Rahman MM, Man AB \& Selama TA (2011) Efficacy and economics of different herbicides in aerobic rice system. African Journal of Biotechnology 10: 8007-8022.

Kasahara Y (1959) Studies on the species of the weeds in Japan and their geographical distribution. Nogaku Kenkyu 47: 123-140. [in Japanese]

Khaliq A, Matloob A, Shafique HM, Cheema ZA \& Wahid A (2011) Evaluating sequential application of pre and post emergence herbicides in dry seeded fine rice. Pakistan Journal of Weed Science Research 17(2): 111-123.

Khaliq A \& Matloob A (2011) Weed-crop competition period in three fine rice cultivars under direct-seeded rice. Pakistan Journal of Weed Science Research 17(3): 229-243.

Kim JK \& Krishnan HB (2002) Making rice a perfect food: Turning dreams into reality. Journal of Crop Production 5: 93-130.

Kumar V\& Ladha JK (2011) Direct seeding of rice: recent developments and future needs. Advances in Agronomy 111: 297-413.

Mabbayad MO, Pablico PP \& Moody K (1983) The effect of time and method of land preparation on weed populations in rice. In: Proceedings of 9th Asian-Pacific Weed Science Society Conference, pp. 357-368.

Maneechote C (2007) Utilization of weeds and their relatives as resources in Thailand. In: Kim KU, Shin DH \& Lee IJ (eds) Utility of weeds and their relatives as resources. Daegu, pp. 107-121.

Mardiyanti ED, Karuniawan PW \& Medha B (2013) Dynamics of plant species diversity post-planting rice. Produksi Tanaman 1(1): 24-35. [in Indonesian]

Marshall EJP, Brown VK, Boatman ND, Lutman PJW, Squire GR \& Ward LK (2003) The role of weeds in supporting biological diversity within crop fields. Weed Research 43: 77-89.

Martin GJ (1995) Ethnobotany - A methods manual. Chapman and Hall, London.

Marcelino LR, Inocencio AI, Zaballa CC \& Paller EC (2005) Bicol's weed recipes. Philippine Journal of Weed Science 23: 40-43.

Michael P (1978) Notes on Echinochloa in the Philippines. Philippine Journal of Weed Science 5: 16-18.

Miles JE, Nishimoto KK \& Kawabata O (1996) Dirurnally altering temperatures stimulate sprouting of purple nut sedge (Cyperus rotundus) tubers. Weed Science 44: 122-125.

Misra MK, Panda A \& Sahu D (2012) Survey of useful wetland plants of south Odisha,India. Indian Journal of Traditional Knowledge 11(4): 658-666.

Mishra N (2016) Ethnomedicinal uses of some bioresources for healing of different ailments in Odisha, India, (Ph. D Thesis). V.K.S. University, Ara.

Mohanty RB, Tripathy BK \& Panda T (2012) Utilization of pith plant (Aeschynomene aspera L., Leguminosae: Papilionoideae) by traditional florists cum craftsmen in Jajpur district, Odisha, India. Nelumbo 54: 168-171.

Moody K (1989) Weeds reported in rice in South and Southeast Asia. Los Baños, 442 p.

Mulungu LS, Jilala MR, Mwatawala MW \& Mwalilino JK (2011) Assesment of damage due to larger grain borer (Prostephanus truncates Horn) on stored paddy rice (Oryza sativa L. Poaceae). Journal of Entomology 8: 295-300.

NatuharaY (2013) Ecosystem services by paddy fields as substitutes of natural wetlands in Japan. Ecological Engineering 56: 97-106.

Noda K (1977) Integrated weed control in rice. In: Freyer JD \& Mastu S (eds) Integrated control of weeds. University of Tokyo Press, Tokyo.

Norgaard RB (1984) Coevolutionary agricultural development. Economic Development \& Cultural Change 32: 525-546.

Nowak A, Sylwia NS \& Marcin NM (2016) Spring weed communities of rice agrocoenoses in central Nepal. Acta Botanica Croatica 75(1): 99-108.

Nyarko KA \& Datta SKD (1993) Effects of light and nitrogen and their interaction on the dynamics of riceweed competition. Weed Research 33: 1-8.

www.tropicalplantresearch.com 
Panda A \& Misra MK (2011) Ethnomedicinal survey of some wetland plants of South Orissa and their conservation. Indian Journal of Traditional Knowledge 10(2): 296-303.

Pane H, Noor ES, Dizon M \& Mortimer AM (2000) Weed communities of gogorancah rice and reflections on management. In: Proceedings of the International Workshop on Characterizing and Understanding Rain fed Environments. Los Banos.

Parameswaran P, Ratheesh NMK \& Kumar AN (2014) Diversity of vascular plants associated with wetland paddy fields (Vayals) of Wayanad District in Western Ghats, India. Annals of Plant Sciences 3(5): 704-714.

Parameswaran P \& Kumar AN (2017) An account of the 'useful weeds' associated with wetland paddy fields (Vayals) of wayanad, Kerala, India. Annals of Plant Sciences 6(1): 1516-1526.

Pemberton RW \& Lee NS (1996) Wild food plants in South Korea; market presence, new crops, and exports to the United States. Economic Botany 50(1): 57-70.

Qiang S (2002) Weed diversity of arable land in China [J]. Journal of Korean Weed Science 22(3): 187-198.

Rao AN, Wani SP, Ahmed SH, Haider Ali H \& Marambe B (2017) An overview of weeds and weed management in rice of South Asia. In: Rao AN \& Matsumoto H (eds) Weed management in rice in the Asian-Pacific region. Asian-Pacific Weed Science Society (APWSS). The Weed Science Society of Japan, Japan and Indian Society of Weed Science, India, pp. 248-281.

Raunkiaer C (1934) The life forms of plants and statistical plant geography. Clarendon Press, Oxford.

Rapoport EH, Raffaele E \& Ghermandi L (1995) Edible weeds: a scarcely used resource. Bulletin of Ecological Society of America 76(3): 163-166.

Samra JS, Singh B \& Kumar K (2003) Managing crop residues in the rice-wheat system of the Indo-Gangetic plain. In: Ladha JK, Hill JE, Duxbury JM, Gupta RK \& Buresh RJ (eds) Improving the productivity and sustainability of rice-wheat systems: Issues and impacts. Madison, pp. 173-195.

Satapathy KB, Sahu BB \& Jena GS (2012) Crop weeds diversity and their ethno- medicinal uses in the treatment of common ailments in Jajpur district of Odisha (India). International Journal of Medicinal and Aromatic Plants 2(1): 80-89.

Saxena HO \& Brahmam M (1996) The Flora of Orissa. Vol. I-IV. Orissa Forest Development Corporation, Bhubaneswar.

Sharma J, Raina AK \& Sharma S (2014) Life form classification and biological spectrum of Lamberi Forest Range, Rajouri, J\&K, India. International Journal of Current Microbiology and Applied Sciences 3(11): 234-23.

Shivakumar KV, Devendra R, Muniswamappa MV, Halesh GK \& Mahadevamurthy M (2014) Weed seed production potentials in Bidens pilosa L. in plantation crops in hill zone of Karnataka. International Journal of Research in Applied, Natural \& Social Sciences 2(2): 11-18.

Singh A, Sharma GP \& Raghubanshi AS (2008) Dynamics of the functional groups in the weed flora of dry land and irrigated agroecosystems in the Gangetic plains of India. Weed Biology and Management 8: 250-259.

Singh J (1974) An Agricultural Atlas of India: Geographical Analysis. Vishal Publications, New Delhi. pp. 156.

Singh Y, Singh B \& Timsina J (2005) Crop residue management for nutrient cyclingand improving soil productivity in rice-based cropping systems in the tropics. Advances in Agronomy 85: 269-407.

Swapna MM, Prakashkumar R, Anoop KP, Manju CN \& Rajith, NP (2011) A review on the medicinal and edible aspects of aquatic and wetland plants of India. Journal of Medicinal Plant Research 5: 7163-7176.

Terry PJ (1976) The Cyperaceae-Still the world's worst weeds? In: Riches CR (eds) The World's Worst Weeds. Farnham, pp. 3-18.

Thakur AS (2015) Floristic composition, life-forms and biological spectrum of tropical dry deciduous forest in Sagar District, Madhya Pradesh, India. Tropical Plant Research 2(2): 112-119.

Tomita S, Nawata E, Kono Y, Inamura T, Nagata Y, Noichana C \& Sributta A (2003a) Impact of direct dry seeding on rain fed paddy vegetation in north-east Thailand. Weed Biology and Management 3: 68-76.

Tomita S, Nawata E, Kono Y, Nagata Y, Noichana C, Sributta A \& InamuraT (2003b) Differences in weed vegetation in response to cultivating methods and water conditions in rainfed paddy fields in north-east Thailand. Weed Biology and Management 3: 117-127.

Tripathy BK, Panda T \& Mohanty RB (2014) Traditional artifacts from 'Bena' grass (Vetiveria zizanioides (L.) Nash., Poaceae) in Jajpur district of Odisha, India. Indian Journal of Traditional Knowledge 12(4): 771-777.

Turki Z \& Sheded M (2002) Some observations on the weed flora of rice fields in the Nile Delta, Egypt. Feddes Repert 113: 394-403.

Turner NJ, Łuczaj ŁJ, Migliorini P, Pieroni A, Dreon AL \& Sacchetti LE (2011) Edible and tended wild plants, 
traditional ecological knowledge and agroecology.Critical Review of Plant Science 30(1\&2): 198-225.

Wassmann R, Jagadish SVK, Sumfleth K, Pathak H, Howell G, Ismail A, Serraj R, Redona E, Singh RK \& Heuer S (2009) Regional vulnerability of climate change impacts on Asian rice production and scope for adaptation. Advances in Agronomy 102: 91-133.

Wills GD (1987) Description of purple and yellow nut sedge (Cyperus rotundus and C. esculentus).Weed Technology 1: 2-9.

Wisler GC \& Norris RE (2005) Interactions between weeds and cultivated plants as related to management of plant pathogens. Weed Science 53: 914-917.

YaherwandiY (2009). Structure of community parasitoids Hymenoptera in various agriculture landscape, West Sumatra. Jurnal Entomologi Indonesia 6 (1):1-14. [in Indonesian]

Yamaguchi H \& Umemoto S (1996) Classification of paddy levees in terms of plant resource complex. Weed Research Japan 41: 286-294. [in Japanese with English summary]

Yu L, Jiang R, Gao Z, Hu S, Li M, Wu M, Yu T \& Chen L (1993) The community and itssuccession of paddy weeds in Zhejiang Province. Weed Science 4: 10-12. [In Chinese]

Yun-He H \& ShengQ (2014) Analysis of farmland weeds species diversity and its changes in the different cropping systems. Bulgarian Journal of Agricultural Science 20(4): 786-794.

Zedler JB \& Kercher S (2005) Wetland resources: status, ecosystem services, degradation, and restorability. Annual Review of Environmental Resources 30: 39-74. 